\title{
Nonlinear dynamics of large-scale activity in "networks of networks"
}

\author{
Fereshteh Lagzi ${ }^{{ }^{*}}$, Fatihcan M Atay ${ }^{2}$, Stefan Rotter ${ }^{1}$ \\ From Twenty Second Annual Computational Neuroscience Meeting: CNS*2013 \\ Paris, France. 13-18 July 2013
}

As a first step toward understanding the macro-dynamics of brain-like systems, we study the large-scale dynamics of balanced random networks of excitatory and inhibitory integrate-and-fire neurons. Based on the dynamical equations of the model, a mean field approach was previously employed to reduce the dimensionality of the network dynamics $[1,2]$. Here, we analyze the joint activity dynamics of excitatory and inhibitory populations employing a pair of mutually interacting nonlinear differential equations. In absence of a voltage leak for individual neurons, and for negligible synaptic transmission delay, these equations take the form of Lotka-Volterra equations. These have been used to describe predatorprey systems, corresponding to excitatory and inhibitory populations of neurons in our case. For non-zero identical synaptic transmission delay, we obtain Lotka-Volterra equations with delay. We try to infer the parameters for the non-autonomous differential equations given a dataset from numerical simulations of such a network. Moreover, we attempt to analytically constrain the parameters and compare them with their statistical estimators. Using simulation results, the significance of the nonlinear dynamics becomes obvious in the vector field of excitatory-inhibitory activity, which corresponds nicely with the vector field of the analytical equations.

We have analyzed the stability of the network considering two bifurcation parameters: the relative strength of recurrent inhibition, "g", which controls the balance between excitation and inhibition in the network, and the intensity of external input to the network, " $\eta$ ". We have found out that for a value of "g" that keeps the exact balance between excitation and inhibition, a bifurcation from unstable to stable network dynamics takes

\footnotetext{
* Correspondence: fereshteh.lagzi@bcf.uni-freiburg.de

'Bernstein Center Freiburg \& Faculty of Biology, University of Freiburg, Germany Full list of author information is available at the end of the article
}

place. This bifurcation separates Synchronous Regular (SR) from Asynchronous Irregular (AI) activity of the network, similar to what was found in a previous study on the same network using a Fokker-Planck approach [3]. The influence of synaptic delays on the reduced dynamics of the network is currently under study.

It has been shown that Lotka-Volterra equations are capable of representing switching dynamics between different states of neural networks $[2,4]$. Our analysis represents a first step toward analyzing the dynamics of more complex "networks of networks" that are implicated in various cognitive abilities of the brain.

\section{Acknowledgements}

This work is supported by the German Federal Ministry of Education and Research (BMBF; grant 01GQ0420 to BCCN Freiburg), the German Research Foundation (DFG; grant EXC 1086 to the Cluster of Excellence BrainLinksBrainTools) and the European Commission (FP7-ICT grant 318723 to MatheMACS).

\section{Author details}

${ }^{1}$ Bernstein Center Freiburg \& Faculty of Biology, University of Freiburg, Germany. ${ }^{2}$ Max Planck Institute for Mathematics in the Sciences Leipzig, Germany.

\section{Published: 8 July 2013}

\section{References}

1. Cardanobile S, Rotter S: Multiplicatively interacting point processes and applications to neural modeling. Journal of Computational Neuroscience 2010, 28(2):267-284.

2. Cardanobile S, Rotter S: Emergent properties of interacting populations of spiking neurons. Frontiers in Computational Neuroscience 2011, 5:59.

3. Brunel N: Dynamics of sparsely connected networks of excitatory and inhibitory spiking neurons. Journal of Computational Neuroscience 2000, 8(3):183-208.

4. Bick C, Rabinovich M: On the occurrence of stable heteroclinic channels in Lotka-Volterra models. Dynamical Systems 2010, 25:97-110.

\section{doi:10.1186/1471-2202-14-S1-P331}

Cite this article as: Lagzi et al:: Nonlinear dynamics of large-scale activity in "networks of networks". BMC Neuroscience 2013 14(Suppl 1): P331.
C Biomed Central

(c) 2013 Lagzi et al; licensee BioMed Central Ltd. This is an Open Access article distributed under the terms of the Creative Commons Attribution License (http://creativecommons.org/licenses/by/2.0), which permits unrestricted use, distribution, and reproduction in any medium, provided the original work is properly cited. 